\title{
Heat shock proteins in stabilization of spontaneously restored sinus rhythm in permanent atrial fibrillation patients after mitral valve surgery
}

\author{
Hailong Cao $\cdot$ Lei Xue $\cdot$ Xiaohan Xu Yanhu Wu • \\ Jinfu Zhu $\cdot$ Liang Chen $\cdot$ Duan Chen $\cdot$ Yijiang Chen
}

Received: 25 February 2011 / Revised: 10 March 2011 / Accepted: 11 March 2011 / Published online: 1 April 2011

(C) The Author(s) 2011. This article is published with open access at Springerlink.com

\begin{abstract}
A spontaneously restored sinus rhythm in permanent atrial fibrillation patients has been often observed after mitral valve (MV) surgery, but persisting duration in sinus rhythm varies from patient to patient. Heat shock proteins (Hsps) may be involved in pathogenesis of atrial fibrillation. We hypothesized that stabilization of restored sinus rhythm is associated with expression of Hsps in the atria. To test this hypothesis, clinical data, biopsies of right atrial appendage, and blood samples were collected from 135 atrial fibrillation patients who spontaneously restored sinus rhythm after conventional isolated MV replacement. Comparison was made between patients who had recurrence of atrial fibrillation within 7 days (AF) vs. patients with persisted sinus rhythm for more than 7 days (SR). Results showed that SR patients had higher activity of heat shock transcription factor 1 (HSF1) as well as upregulated expressions of heat shock cognate 70, Hsp70, and Hsp27 in the tissues. The activation of HSF1-Hsps pathway was associated with less-aggressive pathogenesis as reflected by lower rates of myolysis, apoptosis, interstitial fibrosis, and inflammation in SR patients. However, Hsp60 was lower in both tissue and plasma in SR patients, and was positively correlated with apoptosis, interstitial fibrosis, and inflam-
\end{abstract}

Hailong Cao and Lei Xue contributed equally to this work.

H. Cao $\cdot$ L. Xue $\cdot$ X. Xu $\cdot$ Y. Wu J. Zhu $\cdot$ L. Chen $\cdot$ Y. Chen $(\triangle)$

Department of Thoracic and Cardiovascular Surgery,

The First Affiliated Hospital of Nanjing Medical University,

300 Guangzhou RD,

Nanjing 210029, China

e-mail: YJChen@njmu.edu.cn

H. Cao $\cdot$ D. Chen

Department of Cancer Research and Molecular Medicine,

Norwegian University of Science and Technology,

Trondheim, Norway mation. These findings suggest that the Hsps play important roles in stabilization of restored sinus rhythm after MV surgery by inhibiting AF-related atrial remodeling and arrhythmogenic substrates in atrial fibrillation patients. Low circulating Hsp60 levels preoperatively might predict a stable spontaneously restored sinus rhythm postoperatively.

Keywords Heat shock proteins - Sinus rhythm .

Stabilization · Atrial fibrillation · Mitral valve surgery

\section{Introduction}

Atrial fibrillation (AF) is present in $40-60 \%$ of patients undergoing mitral valve (MV) surgery (Raanani et al. 2001), and has been identified as a risk factor for poor outcome after MV replacement (Ruel et al. 2005). Because of limited success of electrical- and/or drug-induced conversion, concomitant maze procedure and intraoperative radiofrequency ablation have been used during the past two decades (Raanani et al. 2001; Deneke et al. 2007). However, it remains a significant concern for the inevitable risk of perioperative complications and uncertainty regarding atrial mechanical function (Cox et al. 2000; Boyd et al. 2009). In fact, most patients with chronic AF experience a spontaneous restoration of sinus rhythm (SR) after MV surgery. However, some patients could have a persistent SR for a long period of time (from weeks to years), while others might have recurrence of AF shortly after the surgery (usually within 1 week; Chua et al. 1994; Large et al. 1997; Jessurun et al. 2000). The spontaneous restoration of SR may be attributed by an improved transmitral haemodynamics after the surgery (Large et al. 1997; Raine et al. 2004), but the mechanism by which the patients have a persisted SR remains unknown. Moreover, there are no 
reliable biomarkers that could indicate which patients are prone to have the persistent postoperative SR.

Heat shock proteins (Hsps), a family of highly conserved and stress-induced proteins, serve as molecular chaperones to refold denatured proteins and promote the degradation of damaged proteins via the activation and binding of heat shock transcription factor 1 (HSF1) in many physiopathologic processes (Parsell and Lindquist 1993; Sarge et al. 1993). Recently, an elevated expression of Hsps in atrial tissue, including isoforms of Hsp27, heat shock cognate (Hsc) 70, and Hsp60, was found in AF patients (Yang et al. 2007; Brundel et al. 2006a; Schäfler et al. 2002), and higher atrial levels of Hsp70 might be associated with a reduced risk of postoperative AF (Mandal et al. 2005). It has also been shown that induction of Hsps by hyperthermia and/or geranylgeranylacetone could prevent the arrhythmogenic substrates for AF (Takahashi et al. 2009; Brundel et al. 2006b). Conceivably, Hsps play an important role in the pathogenesis and progression of AF.

In the present study, we tested our hypothesis that the stabilization of spontaneously restored SR in patients with permanent AF after MV surgery is associated with expressions of Hsps in atria. To this end, we performed a study by including 135 patients in order to obtain essential clinical data as well as biopsies of right atrial appendages (RAAs) for biochemical and histological analyses of HSF1 activity, Hsps expression, myolysis, apoptosis, interstitial fibrosis, and inflammation (Schotten et al. 2001; Aimé-Sempé et al. 1999; Burstein and Nattel 2008; Aviles et al. 2003). In addition, Hsps levels in the serum were measured, presumably to be used as a marker for AF recurrence.

\section{Methods}

Patients and study design

We consecutively recruited 135 patients admitted to the First Affiliated Hospital of Nanjing Medical University between November 2007 and June 2010. Inclusion criteria were: (1) preoperative permanent AF (persisting at least 1 year; Obadia et al. 1997) and (2) spontaneous restoration of SR after conventional isolated MV replacement surgery. Exclusion criteria were (1) history of cardiac arrhythmia other than AF; (2) need of coronary artery bypass grafting (CABG) or other associated procedures at the same time; (3) requirement of redo surgery; (4) previous permanent pacemaker and/or automatic implantable cardioverter defibrillator implantation; (5) severe postoperative complications; and (6) requirement of postoperative pacemaker implantation or treatment with antiarrhythmic agent (except digitalis) within 7 days. The present study was conducted according to the Helsinki Declaration and approved by the ethics committee of
Nanjing Medical University. All the patients were given written informed consent before being included in the study.

Surgery and tissue collection

The same cardiac anesthesiologist, perfusionist, and surgical team performed all of these operations. All the patients underwent standard cardiopulmonary bypass with moderate hypothermia, antegrade crystalloid cardioplegic arrest, and local hypothermia with ice slash. The MV was approached through a transseptal approach and replaced with prosthetic valves. The 135 patients returned spontaneously to SR when they were weaned from extracorporeal circulation and remained in SR to the end of surgical procedure.

About $250 \mathrm{mg}$ RAA tissue for each patient was obtained from the cannulation site before starting extracorporeal circulation. Fifty milligram RAAs were fixed in paraformaldehyde for histology and immunohistochemistry, and the others were immediately snap-frozen in liquid nitrogen for biochemical analysis.

\section{Recurrence of AF}

After surgery, patients were transferred to the cardiothoracic intensive care unit. The patients were monitored by continuous electrocardiogram (ECG) during a minimum period of $48 \mathrm{~h}$ postoperatively; and afterwards, it was repeated at least every $8 \mathrm{~h}$ for 15 -min each time until discharge. In case of rhythm disturbance reported by nurse or patient in the interval, a 12-lead ECG recording was conducted and continuous ECG monitoring was reused. The recurrence of $\mathrm{AF}$ was diagnosed on the basis of a minimum of 15-min AF duration documented by a continuous 12-lead ECG recording (Mariscalco and Engström 2008). Before discharge, the patients were divided into two groups according to whether (AF group) or not (SR group) AF recurrence took place within 7 days postoperatively (Korantzopoulos et al. 2005).

\section{Western blot}

Frozen RAAs were used for protein isolation as described previously (Brundel et al. 2006b). Proteins (40 $\mu \mathrm{g} / \mathrm{lane})$ were separated by sodium dodecyl sulfate polyacrylamide gel electropheresis and transferred onto polylinylidene fluoride membranes using a Bio-Rad semidry transfer system (Bio-Rad). The membranes were blocked with 5\% non-fat dry milk and then probed with horseradish peroxidase (HRP)-conjugated mouse monoclonal anti-GAPDH (KC5 G5, KangChen Biotech, China), rabbit monoclonal anticleaved caspase-3 (\#9661, Cell Signaling Technology, USA), rabbit polyoclonal anti-HSF1 (SPA-901), mouse monoclonal anti-Hsc70 (ALX-804-067), mouse monoclonal anti-Hsp70 (SPA-810), goat polyoclonal anti-Hsp60 (SPA-828), or rabbit 
polyoclonal anti-Hsp27 (SPA-803; StressGen Biotechnologies, Victoria, Canada). The working dilutions were 1:1,000 for all the primary antibodies. The resulting reaction was visualized using HRP-conjugated anti-rabbit or anti-mouse or anti-goat IgG secondary antibody (Santa-Cruz Biotechnology, the Netherlands), followed by incubation with ECL Western Blot Detection Kit (Amersham, the Netherlands) for $1 \mathrm{~min}$. The blots were exposed to Kodak film for $5 \mathrm{~min}$ and immunoreactive bands developed for quantification using The Discovery Series ${ }^{\mathrm{TM}}$ image analysis software (Bio-Rad) normalized by the corresponding value of GAPDH. Experiments were repeated three times and the mean was scored.

\section{Electrophoretic mobility shift assay}

Preparation of nuclear extracts from RAAs and electrophoretic mobility shift assay (EMSA) were done according to a previous report (Metzler et al. 2003). Briefly, HSF1 binding activity in isolated nuclear protein was examined by EMSA in a $20 \mu \mathrm{l}$ of reaction mixture $[1 \times$ binding buffer, $50 \mathrm{ng} / \mu \mathrm{l}$ poly $(\mathrm{dI} \cdot \mathrm{dC}), 50 \%$ glycerol, $1 \% \mathrm{NP}-40$, $1 \mathrm{M} \mathrm{KCl}, 100 \mathrm{mM} \mathrm{MgCl} 2,15 \mu \mathrm{g}$ of nuclear proteins, and $40 \mathrm{fmol}$ of ${ }^{32} \mathrm{P}$-labeled HSE consensus sequence oligonucleotide (5'-CTAGAAGCTTCTAGAAGCTTCTAG-3')]. The reaction mixture was incubated at room temperature for $20 \mathrm{~min}$ and analyzed by electrophoresis on $6 \%$ nondenaturing polyacrylamide gels. The gels were dried by Gel-drier (Bio-Rad) and visualized by autoradiography. The density of the binding bands was analyzed by the Discovery Series ${ }^{\mathrm{TM}}$ image analysis software (Bio-Rad). The level of activated HSF1 was calculated in relation to the maximum value.

\section{Histology and immunohistochemistry}

After fixation with 4\% paraformaldehyde in phosphatebuffered saline (pH: 7.4) for $24 \mathrm{~h}$, the tissues were subjected to alcoholic dehydration and embedded in paraffin. Two micrometer serial sections were sliced and subjected to Van Gieson staining for microscopic examination. Collagen volume fraction (CVF) was determined by the HPISA 100 chromatic color pathological analysis system using five random images from each slide and five slides per sample, and the mean values of CVF were obtained by one investigator blinded to the groups.

To quantify the degree of myolysis, $2 \mu \mathrm{m}$-thick sections were stained with periodic acid Schiff (PAS) and counterstained with toluidine blue (TB) and examined by light microscopy. At least three sections per patient were examined with a minimum of 200 cells in total. The degree of myolysis was defined according to the percent of severe myolytic myocytes by one investigator who was blinded to the study groups, and mean scores were calculated (Schotten et al. 2001).
Immunohistochemical staining for detecting HSF1, Hsc70, Hsp70, Hsp60, or Hsp27 (the same antibodies as Western blot but with working dilution at $1: 100$ ) was performed on $4 \mu \mathrm{m}$-thick sections from each paraffinembedded tissues, according to a previous report elsewhere (Mandal et al. 2005). A peroxidase-based detection system was used, with diaminobenzidin as the final chromogen.

Terminal-deoxynucleotidyl transferase-mediated nick end labeling

Apoptosis was examined by terminal-deoxynucleotidyl transferase-mediated nick end labeling (TUNEL) method (Aimé-Sempé et al. 1999). Four $4 \mu \mathrm{m}$-thick sections from each block were evaluated for percentage of apoptotic cells using a POD TUNEL kit (Roche). Four fields per section were randomly examined using a defined rectangular field area with magnification $\times 200$. The number of TdT-labeled cells was determined by counting at least 500 myocytes in sections, and expressed as a percentage of the total number of cells.

Immunofluorescent staining

Frozen sections were fixed for $10 \mathrm{~min}$ in $100 \%$ methanol $\left(-20^{\circ}\right.$ C), dried and blocked in 5\% BSA (20 min room temperature). Mouse monoclonal anti-myosin heavy chain (ab15, Abcam, USA) and Hsp27 (the same antibody as immunohistochemistry) were used. The working dilutions were 1:100 for the both primary antibodies. Fluorescein labeled isothiocyanate antimouse (Jackson ImmunoResearch, The Netherlands) or N,N'(dipropyl)-tetramethyl-indocarbocyanine $\mathrm{Cy} 3$ anti-rabbit (Amersham, The Netherlands) was used as secondary antibody. Nuclei were visualized by 4',6-diamidino-2-phenylindole staining.

\section{Enzyme-linked immunosorbent assay}

Venous blood samples were obtained in EDTA from each patient before surgery. Plasma was separated and stored at $-80^{\circ} \mathrm{C}$ until analysis. The concentrations of myocardial tissue interleukin (IL)- 6 and tumor necrosis factor (TNF)- $\alpha$ and plasma Hsps were measured by enzyme-linked immunosorbent assay (ELISA; human TNF- $\alpha$ and IL-10 ELISA kits, Biomed Diagnostics, Singapore; human Hsps ELISA kits, StressGen Biotechnologies, Victoria, Canada), according to the manufacturer's instructions. Assays were performed in triplicate in a single run and normalized to a standard curve.

Statistical analysis

For the comparison between the two groups, Student's $t$ test (normally distributed) or Mann-Whitney test (non-normally 
distributed) was used for continuous variables, and $\chi^{2}$ test was utilized for categorical variables. Correlation analysis (Pearson or Spearman) was used to assess the association between atrial Hsps contents and histological and biochemical results in RAAs and corresponding serum Hsps levels. $p<0.05$ (two-sides) were used as the criterion of statistical significance. Multiple logistic regression analysis was performed to test whether any of the preoperative parameters and plasma Hsps levels could predict early recurrence of AF. All univariate variables with $p<0.1$ were investigated in a multivariate model. In the multivariate model, a variable was excluded when $p \geq$ 0.05 . The statistical analysis was performed with the GBSTAT statistical analysis package (version 9.0, Dynamic Microsystems, Inc).

\section{Results}

\section{Patient characteristics}

The 135 patients were divided into SR group $(n=52)$ and AF group $(n=83)$. No significant differences were found in terms of age and gender distribution. The preoperative echocardiographic atrial sizes were smaller in SR than AF. The preoperative plasma levels of C-reactive protein (CRP) and central venous pressure were lower in SR than AF. In addition, durations of staying at intensive care unit and postoperative hospitalization were shorter in SR than AF (Table 1).

\section{Expression and activity of HSF1 in RAAs}

Immunohistochemistry showed that the expression of HSF1 was comparable between AF and SR groups (Fig. 1a), which was confirmed by Western blot analysis (Fig. 1b,c). To determine whether the modified proportion of HSF1 was larger in SR patients, a small amount of protein $(20 \mu \mathrm{g} /$ lane) was loaded in $10 \%$ gel, which allows better separation of proteins with moderate changes in molecular weight. As shown in Fig. 1d, gray scale of the two HSF1 bands appeared to shift to higher molecular weight species (Dimer and Trimer) and were more intensive in SR than AF. EMSA showed further that the activity of HSF1 was higher in SR than AF (Fig. 1e,f).

\section{Expression of Hsps in RAAs}

Both immunohistochemistry and Western blot analysis showed an increased expression of Hsc70 (1-3 in Fig. 2a), Hsp70 (1-3 in Fig. 2b), and Hsp27 (1-3 in Fig. 2d) and reduced expression of Hsp60 (1-3 in Fig. 2c) in SR than AF. Hsc70 and Hsp70 immunoreactivities were found in both cytoplasm and nucleus (1 in Fig. 2a,b), whereas Hsp60 and Hsp27 were merely found in cytoplasm (1 in Fig. 2c,d).

Myolysis, apoptosis, interstitial fibrosis, and inflammation in RAAs

A degree of myolysis appeared in either AF or SR by PAS and TB staining (Fig. 3a), but the percentage of cells with severe myolysis was found to be lower in SR than AF (Fig. 3b).

Apoptotic cells, revealed by TUNEL method, were less numerous in SR than AF (Fig. 4a,c). Western blot analysis showed further that cleaved caspase- 3 protein levels were also lower in SR than AF (Fig. 4b,d).

Interstitial collagen, revealed by Van Gieson staining and expressed as CVF, was lower in SR than AF (Fig. 5a,b).

The levels of TNF- $\alpha$ and IL- 6 , determined by ELISA, were lower in SR than AF (Fig. 6a,b).

Correlations between atrial Hsps levels and HSF1 activity, myolysis, apoptosis, fibrosis and inflammation in RAAs

Taken these data together, correlation test revealed a positive correlation between HSF1 activity and atrial expressions of Hsc70, Hsp70 and Hsp27, but not Hsp60, and a negative correlation between Hsp27 and myolysis (Table 2). A partial co-localization of Hsp27 with myosin was observed (Fig. 3c). Moreover, ratio of apoptotic cells, cleaved caspase-3 levels and CVF were correlated positively with atrial Hsp60 contents and negatively with atrial Hsc70, Hsp70, and Hsp27 contents. TNF- $\alpha$ and IL-6 levels were correlated positively with atrial Hsp60 contents and negatively with atrial Hsp70 levels. A weak but significant negative correlation was also found between TNF- $\alpha$ and atrial Hsc70 contents ( $p=0.023$; Table 2$)$.

Correlations between plasma and atrial Hsps levels

Plasma levels of Hsc70, Hsp70, Hsp27, and Hsp60 in all the samples were measured by ELISA, and only Hsp60 levels were lower in SR than AF $(p<0.01$; Fig. 7 a; data not shown regarding other Hsps). The correlation test indicated a strong positive correlations between plasma and atrial Hsp60 levels ( $r=0.557, p<0.001$; Fig. $7 b)$.

Preoperative variables predicting early recurrence of $\mathrm{AF}$

The results of multiple logistic regression analysis are shown in Table 3. Preoperative echocardiographic left atrial diameter and plasma CRP and Hsp60 levels were independent predictors of early recurrence of $\mathrm{AF}$ after surgery. 
Table 1 Clinical characteristics of study population
Values are presented as mean \pm SD or number of patients.

$A C E-I$ angiotensin-converting enzyme inhibitor, $C P B$ cardiopulmonary bypass, $E F$ ejection fraction, $L A D$ left atrial diameter, $L V D d$ left ventricular enddiastolic diameter, LVDs left ventricular end-systolic diameter, NYHA New York Heart Association, PASP pulmonary artery systolic pressure, $R A D$ right atrial diameter, TAP tricuspid valve annuloplasty

\begin{tabular}{|c|c|c|c|}
\hline Variables & $\mathrm{AF}$ & SR & $p$ value \\
\hline \multicolumn{4}{|l|}{ Basic data } \\
\hline Patient number $(n)$ & 83 & 52 & - \\
\hline Gender, M/F $(n)$ & $36 / 47$ & $18 / 34$ & 0.312 \\
\hline Age (years) & $48.8 \pm 10.2$ & $49.0 \pm 9.4$ & 0.921 \\
\hline \multicolumn{4}{|l|}{ Preoperative data } \\
\hline Heart rate (beats/min) & $82 \pm 13$ & $82 \pm 13$ & 0.803 \\
\hline Mean artery pressure $(\mathrm{mmHg})$ & $91.7 \pm 11.3$ & $90.2 \pm 8.8$ & 0.858 \\
\hline C-reactive protein $(\mathrm{mg} / \mathrm{L})$ & $5.80 \pm 2.40$ & $3.65 \pm 1.96$ & $<0.001$ \\
\hline Duration of mitral disease (years) & $9.4 \pm 4.1$ & $9.4 \pm 3.7$ & 0.806 \\
\hline Duration of AF (years) & $2.6 \pm 1.5$ & $2.6 \pm 1.5$ & 0.953 \\
\hline NYHA class I/II/III/IV & $3 / 29 / 34 / 17$ & $2 / 16 / 31 / 3$ & 0.067 \\
\hline \multicolumn{4}{|l|}{ Echocardiography } \\
\hline LVDd (mm) & $52.9 \pm 8.2$ & $51.3 \pm 9.6$ & 0.116 \\
\hline LVDs (mm) & $36.4 \pm 6.9$ & $35.8 \pm 8.4$ & 0.283 \\
\hline $\mathrm{EF}(\%)$ & $62.0 \pm 5.8$ & $62.9 \pm 5.2$ & 0.107 \\
\hline $\mathrm{LAD}(\mathrm{mm})$ & $60.8 \pm 8.2$ & $46.9 \pm 5.0$ & $<0.001$ \\
\hline RAD (mm) & $46.3 \pm 5.9$ & $41.4 \pm 3.7$ & $<0.001$ \\
\hline PASP (mmHg) & $41.0 \pm 7.6$ & $38.9 \pm 9.4$ & 0.143 \\
\hline Mitral valve disease $(n)$ & & & 0.144 \\
\hline Pure mitral stenosis & 44 & 28 & \\
\hline Pure mitral regurgitation & 10 & 12 & \\
\hline Mitral stenoregurgitation & 29 & 12 & \\
\hline Cause of mitral valve disease $(n)$ & & & 0.121 \\
\hline Rheumatic & 76 & 43 & \\
\hline Degenerative & 7 & 9 & \\
\hline Preoperative length of stay (days) & $14.2 \pm 6.0$ & $14.8 \pm 4.4$ & 0.297 \\
\hline \multicolumn{4}{|l|}{ Preoperative antiarrhythmics $(n)$} \\
\hline Digitalis & 77 & 42 & 0.036 \\
\hline Calcium-channel blocker & 13 & 3 & 0.084 \\
\hline Beta-blocker & 30 & 12 & 0.110 \\
\hline ACE-I & 24 & 12 & 0.455 \\
\hline \multicolumn{4}{|l|}{ Operative data } \\
\hline Central venous pressure $\left(\mathrm{cmH}_{2} \mathrm{O}\right)$ & $5.1 \pm 1.5$ & $4.3 \pm 1.3$ & $<0.001$ \\
\hline Combined TAP $(n)$ & 56 & 32 & 0.481 \\
\hline Left atrial thrombus $(n)$ & 22 & 9 & 0.216 \\
\hline CPB duration (min) & $97 \pm 11$ & $97 \pm 9$ & 0.901 \\
\hline Aortic clamp time (min) & $56 \pm 9$ & $55 \pm 7$ & 0.504 \\
\hline \multicolumn{4}{|l|}{ Postoperative data } \\
\hline Duration of ventilation $(\mathrm{h})$ & $23.0 \pm 6.0$ & $22.5 \pm 7.4$ & 0.315 \\
\hline Intensive care unit stay $(\mathrm{h})$ & $39.7 \pm 11.9$ & $35.8 \pm 10.4$ & 0.036 \\
\hline Postoperative length of stay (days) & $17.1 \pm 3.2$ & $14.0 \pm 2.5$ & $<0.001$ \\
\hline
\end{tabular}

\section{Discussion}

The present study confirmed that a large preoperative echocardiographic left atrial size was an independent risk factor for early recurrence of AF after MV surgery (Chua et al. 1994; Large et al. 1997; Jessurun et al. 2000). In addition, we also found preoperative higher plasma levels of CRP and Hsp60 could predict early recurrence of AF. More importantly, we further tested the hypothesis that the stabilization of restored SR is associated with expression of Hsps in the atria.

A study using an animal model of atherosclerotic lesions showed that both mechanical stretching and treatment with TNF- $\alpha$ resulted in activation of HSF1, which was associated 
Fig. 1 Expression and activity of heat-shock transcription factor 1 (HSF1) in right atrial appendages (RAAs). a Representative immunostaining photomicrographs of HSF1 $(\times 200$, bar $50 \mu \mathrm{m})$. Note: numerous stained nuclei (red arrows) in SR (right photo). b, c Western blot analysis of atrial HSF1 content. d Western immunoblots of HSF1, separated by $10 \%$ gel with $20 \mu \mathrm{g}$ protein/lane. Note: high dense bands of trimer and dimer in SR. e, f Electrophoretic mobility shift assay of HSE-binding activity of HSF1. NS nonspecific complex. In $\mathbf{c}$ and $\mathbf{f}$, values are expressed as mean $\pm \mathrm{SEM} ;{ }^{*} p<0.01 ; N S$ not significant

with increased Hsps production in the aortas (Metzler et al. 2003). By a combination of genome-wide computational and experimental methods, promoters of Hsps (including Hsp70, Hsc70, Hsp27) are bound by HSF1 (Trinklein et al. 2004). In patients with AF, a higher atrial Hsp70 expression was associated with a lower incidence of postoperative AF (Mandal et al. 2005), and the expressions of Hsc70 and especially Hsp27 in atrial tissues were found to be higher in paroxysmal AF than in persistent AF and SR patients (Yang et al. 2007; Brundel et al. 2006a). Hsp60, which has been recently found the role in inhibiting arrhythmogenic atrial remodeling (De Souza et al. 2010), was reported to be elevated expression in atrial tissues of chronic AF patients in comparison with SR patients (Schäfler et al. 2002). In the present study, we investigated the expression and activity of HSF1 as well as all these Hsps isoforms simultaneously in 135 patients (AF vs. SR). As expected, activation of HSF1 was associated with increased expression of Hsc70, Hsp70 and Hsp27 in SR patients. However, the expression of Hsp60 was unexpectedly reduced and not correlated with the activity of HSF1 in these patients. A study using a rat model of heart failure showed an increased binding of NF-кB but not HSF1 to the binding elements of Hsp60 gene, suggesting that Hsp60 might be not bound by HSF1 (Wang et al. 2010). Moreover, studies using HSF1 knock-out and siRNA-HSF1 mice showed that Hsp60 was unlikely to play an important role in maintaining redox-state, oxidative damage after ischemia-reperfusion (Yan et al. 2002; Yin et al. 2005). Conceivably, the Hsp60 expression is under control by other mechanism rather than HSF1.

Recurrence of AF after cardioversion is likely related to a biologic phenomenon known as remodeling, in which the structural properties of atrial tissue and cardiac cells are progressively and irreversibly altered, creating more favorable substrates for AF (Schotten et al. 2001; Aimé-Sempé et al. 1999; Burstein and Nattel 2008; Aviles et al. 2003). In the present study, we next examined the parameters regarding myolysis, apoptosis, interstitial fibrosis, and inflammation, in which Hsps are known to be involved in general. In our SR patients, the rates of myolysis, apoptosis, interstitial fibrosis, and inflammation in RAAs were lower than that in the AF patients, which were negatively correlated with the expressions of Hsps (i.e., Hsp70, Hsc70, Hsp27) in RAAs. Recently, it was suggested that
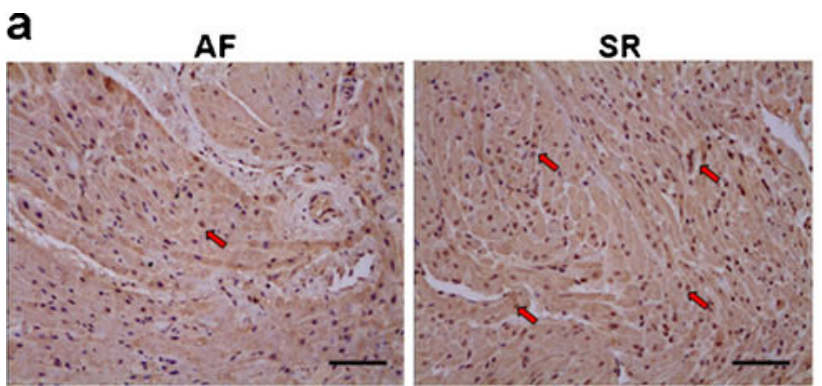

b

AF

SR

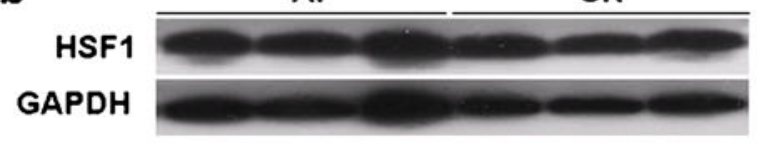

C

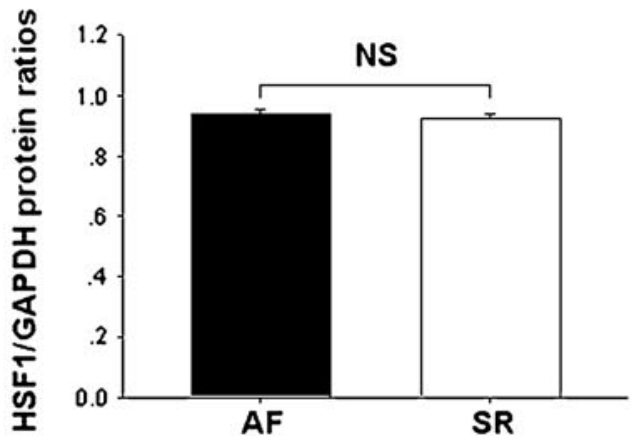

d

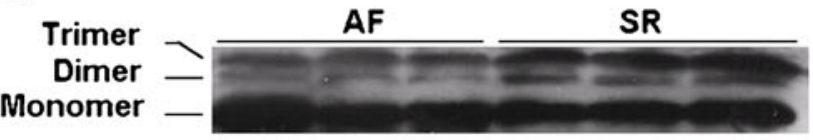

e

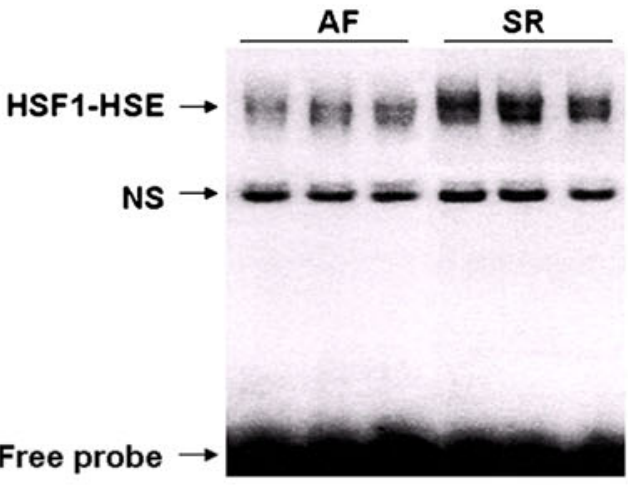

f

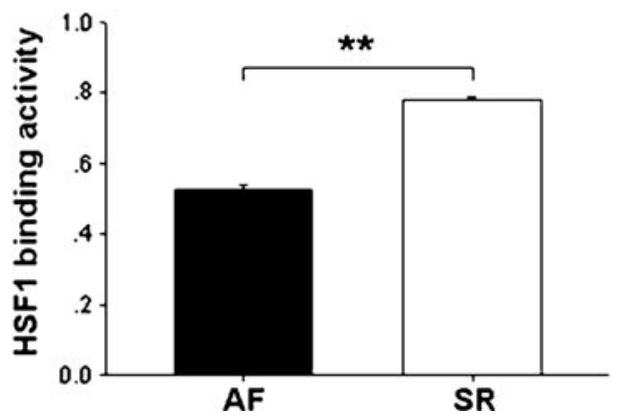



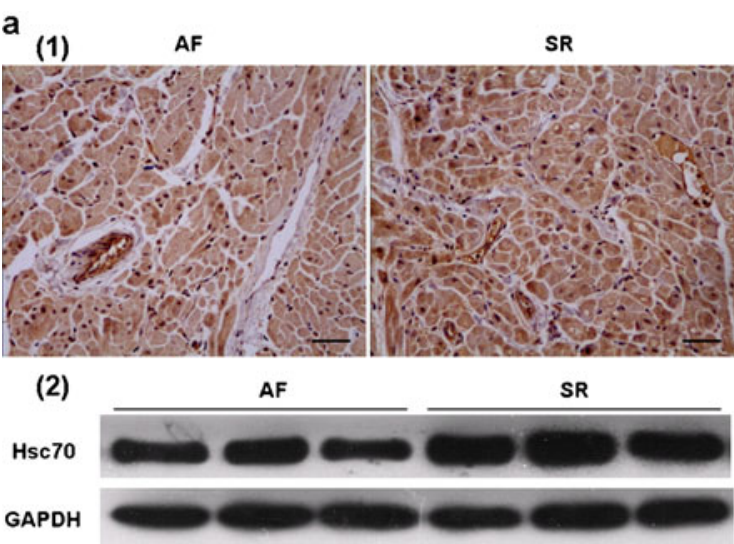

(3)
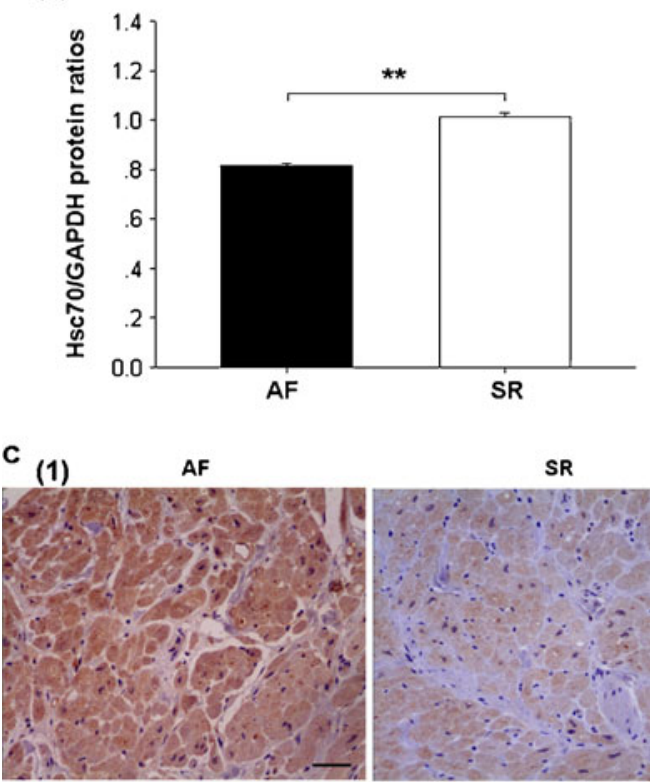

(2)

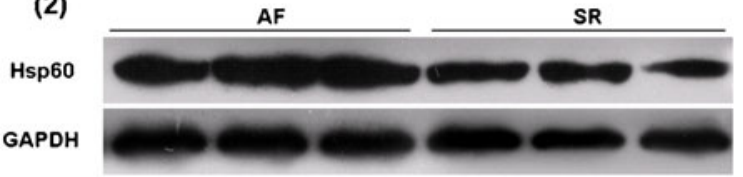

(3)

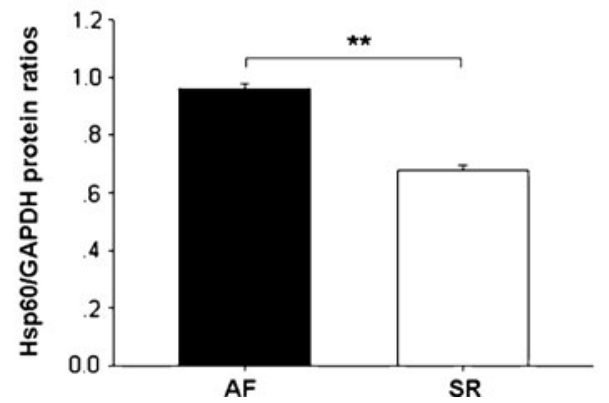

Fig. 2 Expression and localization of heat shock proteins (Hsps) in RAAs: a Hsc70, b Hsp70, c Hsp60, and d Hsp27; 1 representative immunostaining photomicrographs of AF and SR $(\times 200$, bar $50 \mu \mathrm{m})$; b (1)
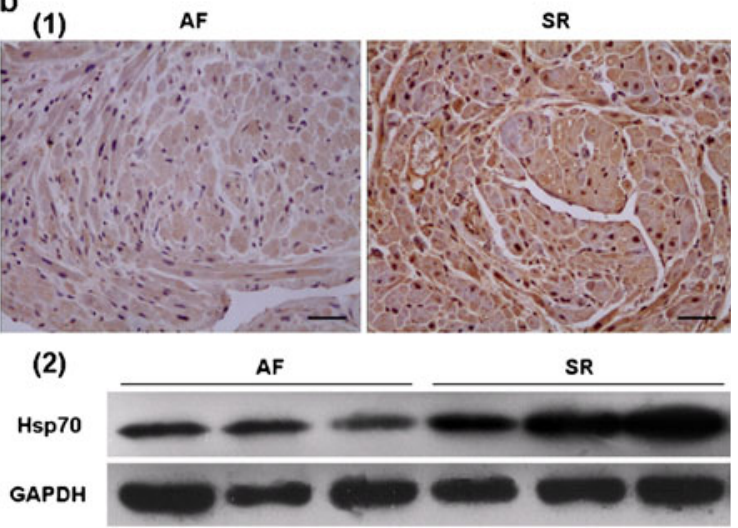

(3)
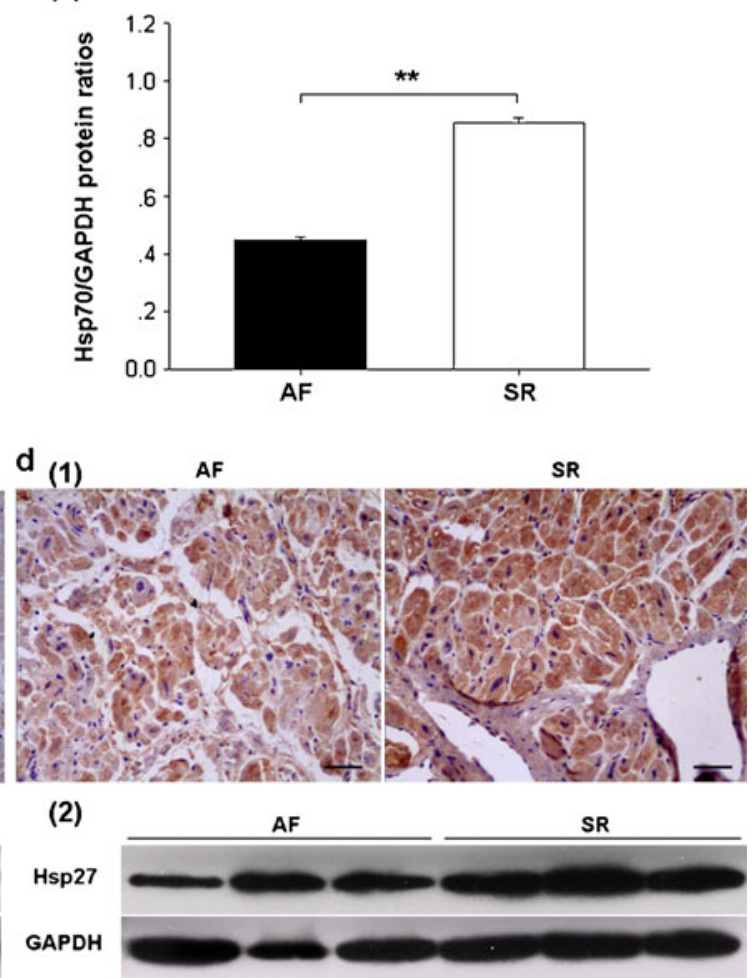

(3)

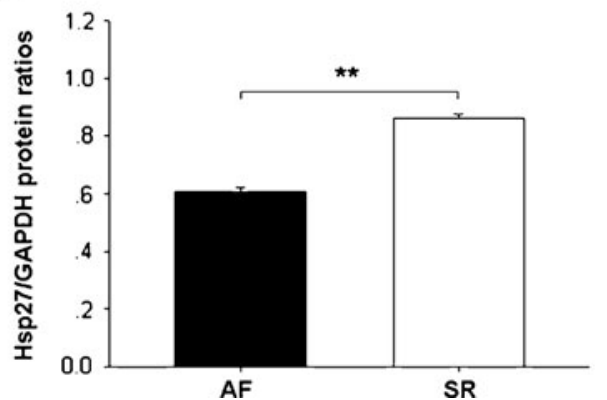

2 and 3 Western blot analysis. Values are expressed as mean $\pm \mathrm{SEM}$; $* * p<0.01$ 
a
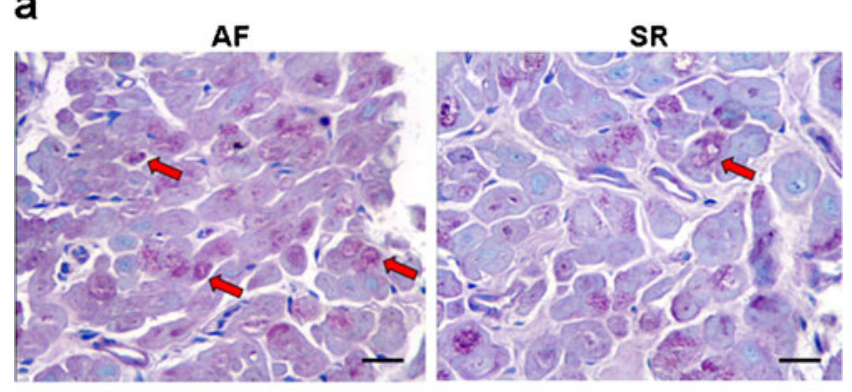

b

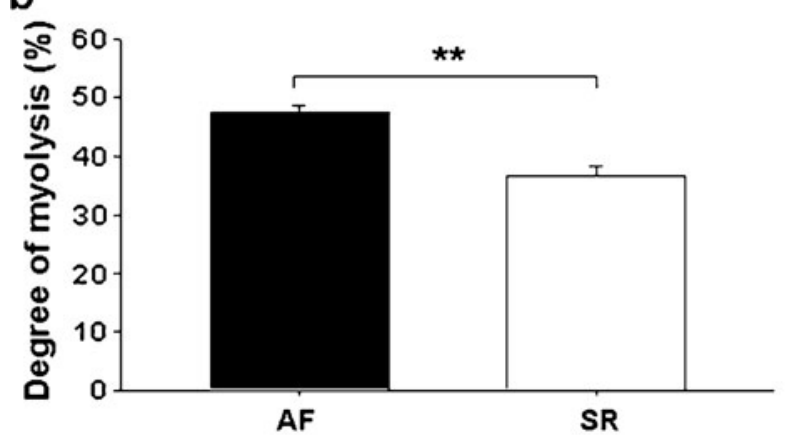

C
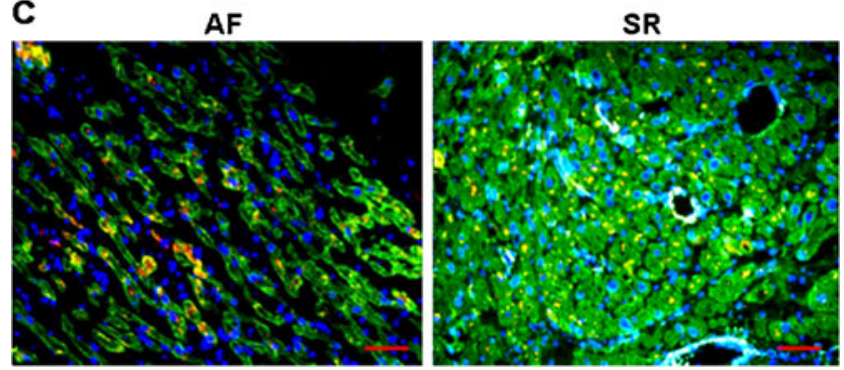

Fig. 3 Myolysis in RAAs and localization of Hsp27 in atrial myofibrils. a Representative photomicrographs showing less loss of sarcomeres (blue staining) and accumulation of glycogen (red staining) in SR (red arrows, $\times 400$, bar $20 \mu \mathrm{m}$ ). b The degree of myolysis, expressed as mean $\pm \mathrm{SEM} ; * * p<0.01$. c Representative immunofluorescent photomicrographs of longitudinal $(A F)$ and transverse $(S R)$ sections showing myosin (green), Hsp27 (red), nuclei (DAPI, blue) and co-localization of Hsp27 with myosin (yellow) in RAAs $(\times 200$, bar $50 \mu \mathrm{m})$

overexpression of $\mathrm{Hsp} 27$ could protect against tachypacinginduced myolysis in a cell model of AF (Brundel et al. 2006a). Indeed, we found that Hsp27 was localized to myofibrils and it was elevated in SR patients.

Sustained AF induces cardiomyocyte apoptosis and fibrosis in the atria, which may promote $\mathrm{AF}$ recurrence after surgery (Aimé-Sempé et al. 1999; Burstein and Nattel 2008). The apoptosis and fibrosis could be attenuated by activation of HSF1 in the heart, as shown in a study using HSF1 transgenic mice (Sakamoto et al. 2006). Indeed, accumulated evidence indicates that the Hsps act directly on the cell death machinery and signaling pathway for cell death (Sreedhar and Csermely 2004). In the present study, we found that the high binding activity of HSF1 and the
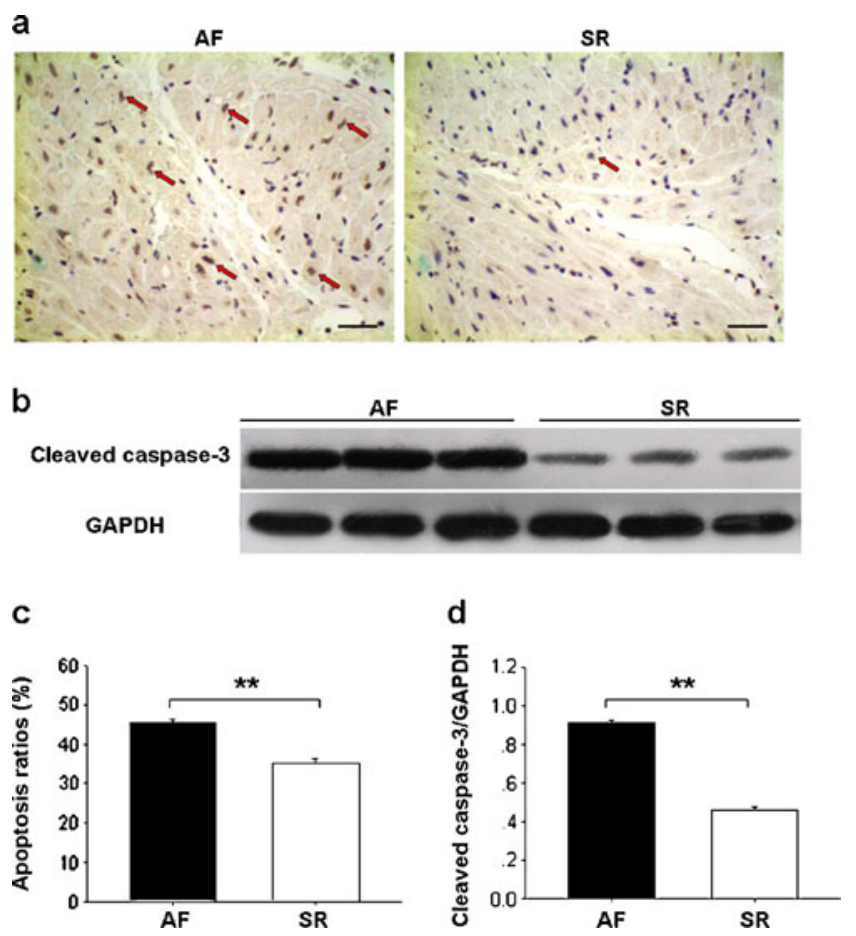

Fig. 4 Apoptosis in RAAs. a Representative photomicrographs of TUNEL staining (red arrows, $\times 200$, bar $50 \mu \mathrm{m}$ ). b Representative Western immunoblots of cleaved caspase-3 and GAPDH. c Apoptosis ratios and $\mathbf{d}$ atrial cleaved caspase- 3 content are expressed as mean \pm $\mathrm{SEM} ;{ }^{*} p<0.01$

\section{a}
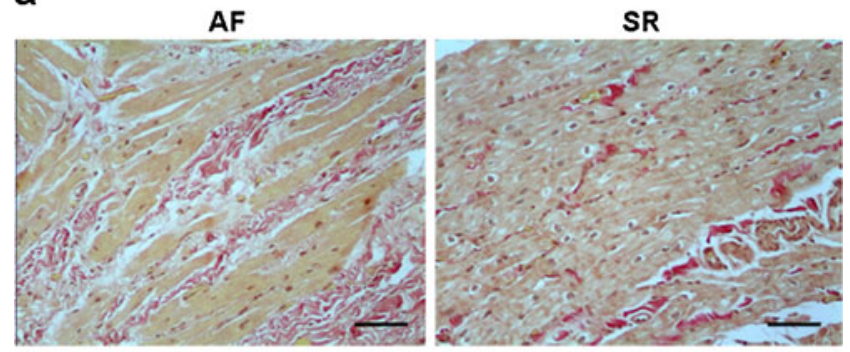

b

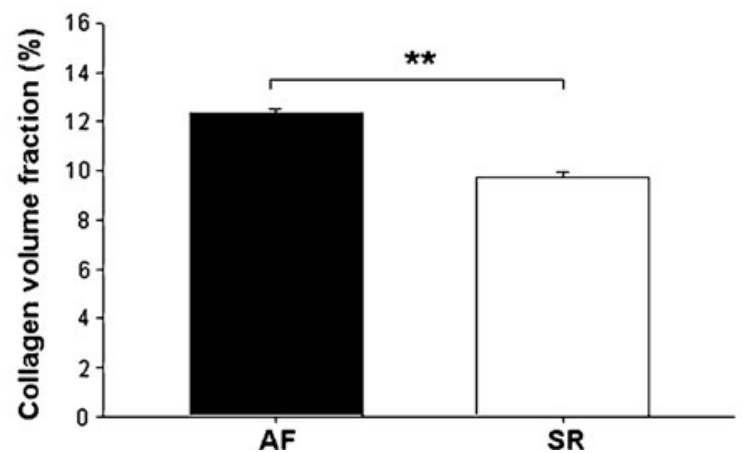

Fig. 5 Fibrosis in RAAs. a Representative photomicrographs of Van Gieson staining showing the interstitial collagen $($ red $)(\times 200$, bar $50 \mu \mathrm{m})$. b Collagen volume fraction is expressed as mean $\pm \mathrm{SEM}$; $* * p<0.01$ 

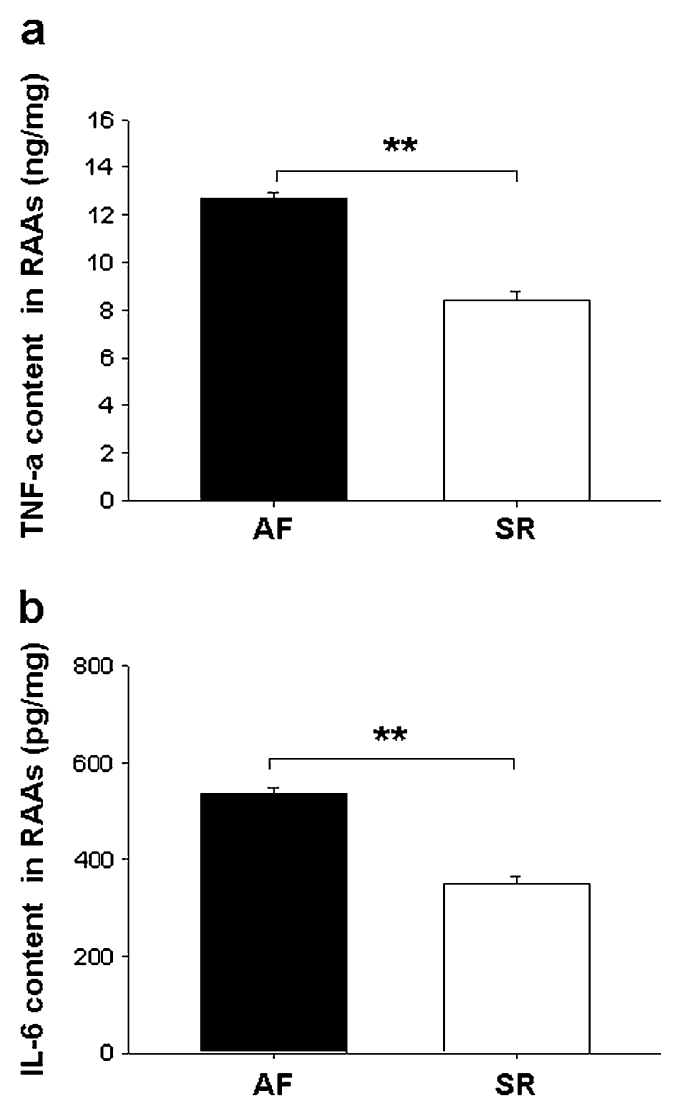

Fig. 6 Inflammation in RAAs. a TNF- $\alpha$ and b IL-6 levels are expressed as mean $\pm \mathrm{SEM} ; * * p<0.01$

increased expressions of Hsc70, Hsp70, and Hsp27 in the atria were associated with the reduced number of apoptotic cells as well as levels of cleaved caspase-3 in SR patients, suggesting that the reduced caspase-3-mediated apoptosis by the activation of HSF1-Hsps pathway may attribute to the stabilization of restored SR. Furthermore, Hsps, especially Hsp70, Hsp27 and Hsc70, have been shown to exhibit anti-fibrotic effects (Takahashi et al. 2009; Wakisaka et al. 2007; Zou et al. 2008; Chen et al. 2004). In the present study, we found that the same HSF1-Hsps pathway of fibrosis as the apoptosis may also play a role in stabilization of restored SR.
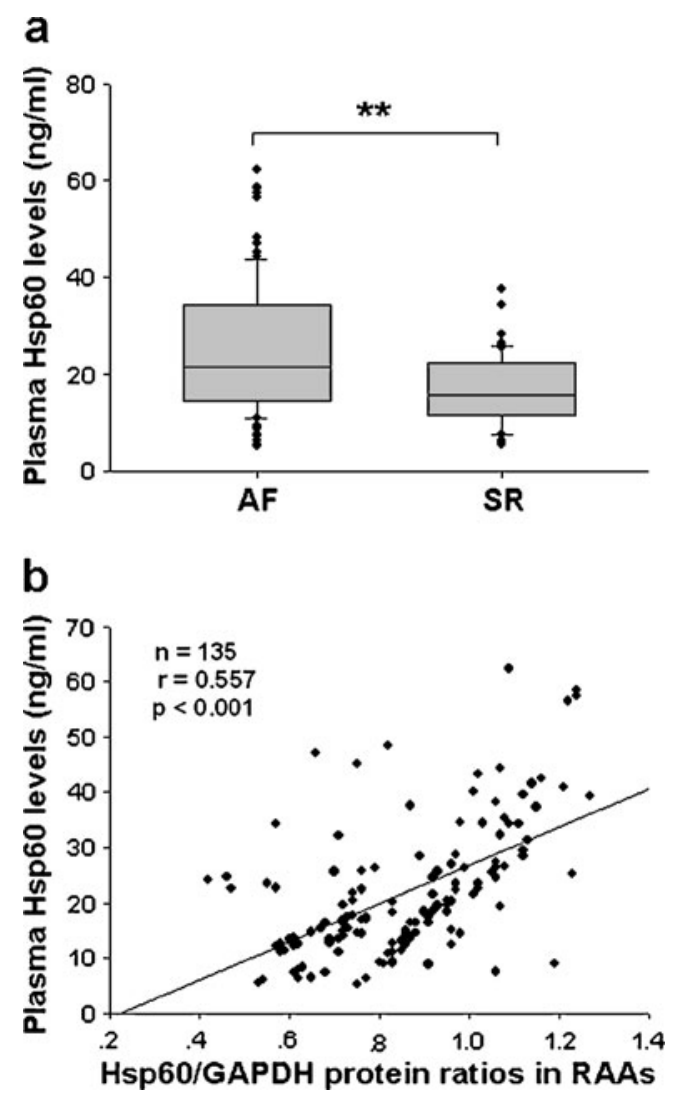

Fig. 7 Correlation between plasma levels and atrial contents of Hsp60. a Box plots illustrating median plasma levels of Hsp60 in AF and SR. Boxes interquartile ranges, bars 10th and 90th percentiles. $* * p<0.01$. b Correlation test

Inflammation has been suggested to play an important role in atrial remodeling, which encourages the propensity to relapse into AF after cardioversion (Aviles et al. 2003; Korantzopoulos et al. 2005; Dernellis and Panaretou 2004). The expression of Hsp70 is elevated in response to inflammation (Chen et al. 2004), and moderate hyperthermia during cardiopulmonary bypass could attenuate the inflammatory response by inducing expression of Hsp70 (Hayashi et al. 2002). Pretreatment with Hsc70 both in vitro and in
Table 2 Correlations between atrial Hsps levels and HSF1 activity, myolysis, apoptosis, interstitial fibrosis and inflammation in RAAs

CVF collagen volume fraction ${ }^{*} p<0.05,{ }^{* *} p<0.01$

\begin{tabular}{|c|c|c|c|c|}
\hline & \multicolumn{4}{|c|}{ Correlation coefficient } \\
\hline & Hsc70 & Hsp70 & Hsp60 & Hsp27 \\
\hline HSF1 activity & $0.620^{* *}$ & $0.766^{* *}$ & -0.211 & $0.650^{* *}$ \\
\hline Degree of myolysis & -0.073 & -0.114 & 0.204 & $-0.512^{* *}$ \\
\hline Ratio of apoptotic cell & $-0.634^{* *}$ & $-0.758^{* *}$ & $0.537^{* *}$ & $-0.420^{* *}$ \\
\hline Cleaved caspase- 3 & $-0.741^{* *}$ & $-0.890^{* *}$ & $0.655^{* *}$ & $-0.569^{* *}$ \\
\hline CVF & $-0.607^{* *}$ & $-0.732^{* *}$ & $0.467^{* *}$ & $-0.528^{* *}$ \\
\hline TNF- $\alpha$ & $-0.322^{*}$ & $-0.416^{* *}$ & $0.612^{* *}$ & -0.220 \\
\hline IL-6 & -0.218 & $-0.403^{* *}$ & $0.582^{* *}$ & -0.177 \\
\hline
\end{tabular}


Table 3 Preoperative predictors of AF early recurrence in multivariate logistic regression analysis

\begin{tabular}{lrrr}
\hline Variables & \multicolumn{1}{l}{ 95\%CI } & $p$ value \\
\hline C reactive protein & -0.043 & -0.067 to -0.018 & 0.001 \\
LAD & -0.029 & -0.035 to -0.023 & $<0.001$ \\
RAD & -0.005 & -0.017 to 0.008 & 0.445 \\
NYHA class & 0.025 & -0.054 to 0.104 & 0.538 \\
Central venous pressure & 0.013 & -0.031 to 0.057 & 0.564 \\
Plasma Hsp60 & -0.007 & -0.012 to -0.002 & 0.006 \\
\hline
\end{tabular}

$B$ regression coefficient, $C I$ confidence interval; other abbreviations as in Table 1

vivo was also shown to inhibit the production of TNF- $\alpha$ (Zou et al. 2008; Su et al. 2010). In the present study, reduced levels of TNF- $\alpha$ and IL-6 in the atrial tissue of SR patients were found, which was in line with the report showing elevated levels of TNF- $\alpha$ and IL-6 in AF patients (Qu et al. 2009).

Unlike other Hsps, Hsp60 expression was found to be positively correlated with apoptosis, interstitial fibrosis and inflammation in RAAs. A recent study in cardiac myocytes showed that Hsp60 activated Toll-like receptor 4, leading to cytokine production and myocyte apoptosis (Kim et al. 2009). A study in human myocardial specimens with heart failure showed also that Hsp60 was positively correlated with apoptosis (Lin et al. 2007). It is known that TNF- $\alpha$ increases the myocyte apoptosis as well as Hsp60 expression, probably by activation of NF-KB and that Hsp60 contains NF-KB binding elements (Kim et al. 2009). In a study with rat model of heart failure, an increased binding of NF-KB to both binding elements of Hsp60 gene was suggested to be associated with enhanced levels of inflammatory factors (Wang et al. 2010). Furthermore, in a cardiac cell line, TNF$\alpha$ treatment increased the Hsp60 expression, which could be prevented by pretreatment with siRNA inhibiting p65 expression (Wang et al. 2010). Taken together, we may suggest that reduced expression of Hsp60 in SR patients is partly associated with reduced rate of apoptosis and resultant interstitial fibrosis and digression of inflammation. We therefore propose that autologous Hsp60 may serve as a deleterious agent for the stabilization of restored SR.

Hsps have been considered to function intracellularly (Parsell and Lindquist 1993; Mandal et al. 2005; Brundel et al. 2006b). Recently, it has been suggested that these proteins can also be released from cells and present in the peripheral blood (Pockley et al. 2003; Zhang et al. 2008). According to previous studies, increased preoperative plasma anti-Hsp60 IgG levels and enhanced pre- and postoperative circulating Hsp70 levels in patients undergoing CABG were associated with the occurrence of postoperative AF (Oc et al. 2007, 2008). The mechanisms were considered to the possible role of Hsps in inflammation and cross-reactive autoimmunity during the development of AF. In contrast, Mandal et al. observed that high intracellular, but not circulating Hsp70 was associated with postoperative AF, and they also failed to identify a correlation between atrial Hsp70 content and serum-soluble Hsp70 level (Mandal et al. 2005). In the present study, we found, for the first time, plasma Hsp60 levels, but not Hsc70, Hsp70, and Hsp27, could reflect its atrial contents and could predict early recurrence of $\mathrm{AF}$ independently. Therefore, we suggest that the determination of plasma levels of Hsp60 before surgery could be used to predict the stabilization of restored SR after the surgery.

Potential clinical perspective

Activation of HSF1-Hsps pathway by hyperthermia and/or geranylgeranylacetone may prevent the arrhythmogenic substrates for AF (Takahashi et al. 2009; Brundel et al. 2006b). Because the stabilization of restored SR after MV surgery is associated with activation of Hsp70, Hsp27, and Hsc70, interventions that stimulate these proteins may prevent the atrial remodeling that leads to AF development and recurrence, thereby affording a new possible therapeutic modality in managing this common complication.

Although successful restoration of SR has been identified to increase quality of life and survival (Raanani et al. 2001; Deneke et al. 2007), the results of surgical cardioversion were controversial in MV patients with permanent AF (Chua et al. 1994; Large et al. 1997; Jessurun et al. 2000; Raine et al. 2004). The patients with early recurrence of AF have more advanced pathological changes in the atria and may be good candidates for concomitant anti-AF procedures. Therefore, it is valuable in predicting which patients in $\mathrm{AF}$ undergoing MV surgery are prone to having the early recurrence of $\mathrm{AF}$ postoperatively. According to the present study, we may suggest that the patients with larger preoperative echocardiographic left atrial sizes, higher preoperative plasma levels of CRP and especially Hsp60 are more likely to develop an early recurrence and are suitable for a concomitant surgical cardioversion.

\section{Study limitations}

First, we could not exclude the patients who had asymptomatic recurrences of AF for more than 15 min but spontaneously restored SR in the interval of 12-lead ECG monitoring at least every $8 \mathrm{~h}$. Therefore, it was possible that some asymptomatic early recurrence occur. Second, owing to a lack of information regarding left atria, it is unknown if the left atria could be a more ideal material in the patients studied, thus further evaluation is warranted. Third, the underlying mechanisms of the reduction of Hsp60 in both tissue and circulation in SR patients need to be elucidated in future studies. Finally, a large 
prospective case-control trial is warranted to calculate the sensitivity, specificity, and predictive values of circulating Hsp60.

\section{Conclusions}

A significant activation of HSF1 and resultant accumulation of Hsc70, Hsp70, and Hsp27 in atrial tissues may prevent the recurrence of $\mathrm{AF}$ in patients after conventional isolated $\mathrm{MV}$ replacement. It is likely that these Hsps could stabilize the restored SR by inhibiting AF-related atrial remodeling and substrates of pathogenesis. Exceptionally, Hsp60 might play adverse roles in those patients. Preoperative low circulating Hsp60 levels may indicate a stable SR postoperatively.

Acknowledgments This work was supported in part by the National Natural Science Foundation of China [30876324]; Jiangsu Province Natural Science Foundation [BK2006248] and a grant for the PhD candidates of Jiangsu Province [JX22013082]. The authors thank all individuals who voluntarily participated in the study and Dr. Hongtai Ma (Department of Pathology, the First Affiliated Hospital of Nanjing Medical University, Nanjing, China) for his technical assistance in special stainings and Dr. Yongyue Wei (Department of Biostatistics, Nanjing Medical University, Nanjing, China) for his statistical assistance.

\section{Conflict of interest None}

Open Access This article is distributed under the terms of the Creative Commons Attribution Noncommercial License which permits any noncommercial use, distribution, and reproduction in any medium, provided the original author(s) and source are credited.

\section{References}

Aimé-Sempé C, Folliguet T, Rücker-Martin C, Krajewska M, Krajewska S, Heimburger M, Aubier M, Mercadier JJ, Reed JC, Hatem SN (1999) Myocardial cell death in fibrillating and dilated human right atria. J Am Coll Cardiol 34:1577-1586

Aviles RJ, Martin DO, Apperson-Hansen C, Houghtaling PL, Rautaharju P, Kronmal RA, Tracy RP, Van Wagoner DR, Psaty BM, Lauer MS, Chung MK (2003) Inflammation as a risk factor for atrial fibrillation. Circulation 108:3006-3010

Boyd AC, Schiller NB, Ross DL, Thomas L (2009) Differential recovery of regional atrial contraction after restoration of sinus rhythm after intraoperative linear radiofrequency ablation for atrial fibrillation. Am J Cardiol 103:528-534

Brundel BJ, Henning RH, Ke L, van Gelder IC, Crijns HJ, Kampinga $\mathrm{HH}$ (2006a) Heat shock protein upregulation protects against pacing-induced myolysis in HL-1 atrial myocytes and in human atrial fibrillation. J Mol Cell Cardiol 41:555-562

Brundel BJ, Shiroshita-Takeshita A, Qi X, Yeh YH, Chartier D, van Gelder IC, Henning RH, Kampinga HH, Nattel S (2006b) Induction of heat shock response protects the heart against atrial fibrillation. Circ Res 99:1394-1402

Burstein B, Nattel S (2008) Atrial fibrosis: mechanisms and clinical relevance in atrial fibrillation. J Am Coll Cardiol 51:802-809
Chen Y, Arrigo AP, Currie RW (2004) Heat shock treatment suppresses angiotensin II-induced activation of NF-kappaB pathway and heart inflammation: a role for IKK depletion by heat shock? Am J Physiol Heart Circ Physiol 287:H1104H1114

Chua YL, Schaff HV, Orszulak TA, Morris JJ (1994) Outcome of mitral valve repair in patients with preoperative atrial fibrillation. Should the maze procedure be combined with mitral valvuloplasty? J Thorac Cardiovasc Surg 107:408-415

Cox JL, Ad N, Palazzo T, Fitzpatrick S, Suyderhoud JP, DeGroot KW, Pirovic EA, Lou HC, Duvall WZ, Kim YD (2000) Current status of the Maze procedure for the treatment of atrial fibrillation. Semin Thorac Cardiovasc Surg 12:15-19

De Souza AI, Cardin S, Wait R, Chung YL, Vijayakumar M, Maguy A, Camm AJ, Nattel S (2010) Proteomic and metabolomic analysis of atrial profibrillatory remodelling in congestive heart failure. J Mol Cell Cardiol 49:851-863

Deneke T, Khargi K, Lemke B, Lawo T, Lindstaedt M, Germing A, Brodherr T, Bösche L, Mügge A, Laczkovics A, Grewe PH, Fritz M (2007) Intra-operative cooled-tip radiofrequency linear atrial ablation to treat permanent atrial fibrillation. Eur Heart J 28:2909-2914

Dernellis J, Panaretou M (2004) Relationship between C-reactive protein concentrations during glucocorticoid therapy and recurrent atrial fibrillation. Eur Heart J 25:1100-1107

Hayashi Y, Sawa Y, Fukuyama N, Nakazawa H, Matsuda H (2002) Preoperative glutamine administration induces heat-shock protein 70 expression and attenuates cardiopulmonary bypass-induced inflammatory response by regulating nitric oxide synthase activity. Circulation 106:2601-2607

Jessurun ER, van Hemel NM, Kelder JC, Elbers S, de la Rivière AB, Defauw JJ, Ernst JM (2000) Mitral valve surgery and atrial fibrillation: is atrial fibrillation surgery also needed? Eur $\mathrm{J}$ Cardiothorac Surg 17:530-537

Kim SC, Stice JP, Chen L, Jung JS, Gupta S, Wang Y, Baumgarten G, Trial J, Knowlton AA (2009) Extracellular heat shock protein 60, cardiac myocytes, and apoptosis. Circ Res 105:1186-1195

Korantzopoulos P, Kolettis TM, Kountouris E, Dimitroula V, Karanikis P, Pappa E, Siogas K, Goudevenos JA (2005) Oral vitamin $\mathrm{C}$ administration reduces early recurrence rates after electrical cardioversion of persistent atrial fibrillation and attenuates associated inflammation. Int J Cardiol 102:321-326

Large SR, Hosseinpour AR, Wisbey C, Wells FC (1997) Spontaneous cardioversion and mitral valve repair: a role for surgical cardioversion (Cox-maze)? Eur J Cardiothorac Surg 11:76-80

Lin L, Kim SC, Wang Y, Gupta S, Davis B, Simon SI, Torre-Amione G, Knowlton AA (2007) HSP60 in heart failure: abnormal distribution and role in cardiac myocyte apoptosis. Am J Physiol Heart Circ Physiol 293:H2238-2247

Mandal K, Torsney E, Poloniecki J, Camm AJ, Xu Q, Jahangiri M (2005) Association of high intracellular, but not serum, heat shock protein 70 with postoperative atrial fibrillation. Ann Thorac Surg 79:865-871

Mariscalco G, Engström KG (2008) Atrial fibrillation after cardiac surgery: risk factors and their temporal relationship in prophylactic drug strategy decision. Int J Cardiol 129:354-362

Metzler B, Abia R, Ahmad M, Wernig F, Pachinger O, Hu Y, Xu Q (2003) Activation of heat shock transcription factor 1 in atherosclerosis. Am J Pathol 162:1669-1676

Obadia JF, el Farra M, Bastien OH, Lièvre M, Martelloni Y, Chassignolle JF (1997) Outcome of atrial fibrillation after mitral valve repair. J Thorac Cardiovasc Surg 114:179-185

Oc M, Ucar HI, Pinar A, Akbulut B, Oc B, Akinci SB, Akyon Y, Kanbak M, Boke E, Dogan R (2007) Heat shock protein 60 antibody: a new marker for subsequent atrial fibrillation development. Saudi Med J 28:844-847 
Oc M, Ucar HI, Pinar A, Akbulut B, Oc B, Akyon Y, Kanbak M, Dogan R (2008) Heat shock protein70: a new marker for subsequent atrial fibrillation development? Artif Organs 32:846-850

Parsell DA, Lindquist S (1993) The function of heat-shock proteins in stress tolerance: degradation and reactivation of damaged proteins. Annu Rev Genet 27:437-496

Pockley AG, Georgiades A, Thulin T, de Faire U, Frostegård J (2003) Serum heat shock protein 70 levels predict the development of atherosclerosis in subjects with established hypertension. Hypertension 42:235-238

Qu YC, Du YM, Wu SL, Chen QX, Wu HL, Zhou SF (2009) Activated nuclear factor-kappaB and increased tumor necrosis factor-alpha in atrial tissue of atrial fibrillation. Scand Cardiovasc J 43:292-297

Raanani E, Albage A, David TE, Yau TM, Armstrong S (2001) The efficacy of the Cox/maze procedure combined with mitral valve surgery: a matched control study. Eur J Cardiothorac Surg 19:438-442

Raine D, Dark J, Bourke JP (2004) Effect of mitral valve repair/ replacement surgery on atrial arrhythmia behavior. J Heart Valve Dis 13:615-621

Ruel M, Kulik A, Lam BK, Rubens FD, Hendry PJ, Masters RG, Bédard P, Mesana TG (2005) Long-term outcomes of valve replacement with modern prostheses in young adults. Eur J Cardiothorac Surg 27:425-433

Sakamoto M, Minamino T, Toko H, Kayama Y, Zou Y, Sano M, Takaki E, Aoyagi T, Tojo K, Tajima N, Nakai A, Aburatani H, Komuro I (2006) Upregulation of heat shock transcription factor 1 plays a critical role in adaptive cardiac hypertrophy. Circ Res 99:1411-1418

Sarge KD, Murphy SP, Morimoto RI (1993) Activation of heat shock gene transcription by heat shock factor-1 involves oligomerization, acquisition of DNA-binding activity, and nuclear localization and can occur in the absence of stress. Mol Cell Biol 13:1392-1407

Schäfler AE, Kirmanoglou K, Balbach J, Pecher P, Hannekum A, Schumacher B (2002) The expression of heat shock protein 60 in myocardium of patients with chronic atrial fibrillation. Basic Res Cardiol 97:258-261

Schotten U, Ausma J, Stellbrink C, Sabatschus I, Vogel M, Frechen D, Schoendube F, Hanrath P, Allessie MA (2001) Cellular mechanisms of depressed atrial contractility in patients with chronic atrial fibrillation. Circulation 103:691-698
Sreedhar AS, Csermely P (2004) Heat shock proteins in the regulation of apoptosis: new strategies in tumor therapy: a comprehensive review. Pharmacol Ther 101:227-257

Su X, Sykes JB, Ao L, Raeburn CD, Fullerton DA, Meng X (2010) Extracellular heat shock cognate protein 70 induces cardiac functional tolerance to endotoxin: differential effect on TNFalpha and ICAM-1 levels in heart tissue. Cytokine 51:60-66

Takahashi N, Wakisaka O, Yoshimatsu H, Saikawa T (2009) Induction of heat shock proteins prevents the arrhythmogenic substrate for atrial fibrillation. Int J Hyperthermia 25:641-646

Trinklein ND, Murray JI, Hartman SJ, Botstein D, Myers RM (2004) The role of heat shock transcription factor 1 in the genome-wide regulation of the mammalian heat shock response. Mol Biol Cell 15:1254-1261

Wakisaka O, Takahashi N, Shinohara T, Ooie T, Nakagawa M, Yonemochi H, Hara M, Shimada T, Saikawa T, Yoshimatsu H (2007) Hyperthermia treatment prevents angiotensin II-mediated atrial fibrosis and fibrillation via induction of heat-shock protein 72. J Mol Cell Cardiol 43:616-626

Wang Y, Chen L, Hagiwara N, Knowlton AA (2010) Regulation of heat shock protein 60 and 72 expression in the failing heart. J Mol Cell Cardiol 48:360-366

Yan LJ, Christians ES, Liu L, Xiao X, Sohal RS, Benjamin IJ (2002) Mouse heat shock transcription factor 1 deficiency alters cardiac redox homeostasis and increases mitochondrial oxidative damage. EMBO J 21:5164-5172

Yang M, Tan H, Cheng L, He M, Wei Q, Tanguay RM, Wu T (2007) Expression of heat shock proteins in myocardium of patients with atrial fibrillation. Cell Stress Chaperones 12:142-150

Yin C, Xi L, Wang X, Eapen M, Kukreja RC (2005) Silencing heat shock factor 1 by small interfering RNA abrogates heat shockinduced cardioprotection against ischemia-reperfusion injury in mice. J Mol Cell Cardiol 39:681-689

Zhang X, He M, Cheng L, Chen Y, Zhou L, Zeng H, Pockley AG, Hu FB, Wu T (2008) Elevated heat shock protein 60 levels are associated with higher risk of coronary heart disease in Chinese. Circulation 118:2687-2693

Zou N, Ao L, Cleveland JC Jr, Yang X, Su X, Cai GY, Banerjee A, Fullerton DA, Meng X (2008) Critical role of extracellular heat shock cognate protein 70 in the myocardial inflammatory response and cardiac dysfunction after global ischemiareperfusion. Am J Physiol Heart Circ Physiol 294:H2805-H2813 